\title{
Bronchoscopy in Bronchiectasis and Cystic Fibrosis
}

\author{
Aditya Kasarabada, Mark E. Lund and Jeffrey B. Hoag \\ Cancer Treatment Centers of America, \\ Drexel University College of Medicine, Philadelphia, PA
}

USA

\section{Introduction}

Bronchiectasis is a constellation of diseases characterized by abnormally dilated bronchi with thickened bronchial walls due to repeated infection and inflammation. Bronchiectasis causes impairment of mucociliary clearance, airflow limitation, bronchorrhea, and predisposes to recurrent respiratory infections. It has a number of potential underlying causes. Laennec first described bronchiectasis as a distinct clinical entity in 1819 (Barker 2002, O'Donnell 1998). The diagnosis, investigation and particularly management of bronchiectasis has been largely empirical and unfortunately, the subject of relatively few controlled clinical trials. Cystic fibrosis causes about a third of all bronchiectasis in United States (O'Donnell 1998), and is common worldwide. Cystic fibrosis (CF) is a recessive genetic disease characterized by dehydration of the airway surface liquid and impaired mucociliary clearance caused by altered functioning of a chloride channel called the Cystic Fibrosis Transmembrane Conductance Regulator (CFTR). Impaired chloride conductance through the apical portion of airway cells leads to dehydration of airway secretions causing lung destruction through obstruction of the airways with thickened secretions. The resultant endobronchial infection and exaggerated inflammatory response leads to the development of bronchiectasis (destruction and widening of airways) and progressive obstructive airway disease. This chapter provides insight into the specific diagnostic and therapeutic roles of bronchoscopy in patients with bronchiectasis and Cystic Fibrosis.

\section{Overview of bronchiectasis}

Bronchiectasis is generally defined as an abnormal and permanent dilatation of the bronchi with thickening of the bronchial wall. In a retrospective cohort study of the insurance claims made in United States, Weycker et al. showed the prevalence of bronchiectasis ranged from 4.2 per 100,000 persons aged $18-34$ years to 271.8 per 100,000 among those aged $\geq 75$ years. Prevalence is higher among women than men at all ages (Weycker, D 2005). Bronchiectasis is being recognized with increasing frequency because of the widespread use of highresolution chest computed tomography (HRCT) scanning (Cohen, M 1999). A bronchus is thought to be dilated if on the CT scan the broncho-arterial ratio with the adjacent accompanying artery exceeds 1 . There are many causes of bronchiectasis that can be classified as due to anatomic, systemic diseases, congenital, post infectious, or idiopathic 
(Barker, A.F. 2002, O'Donnell, A.E. 2008). Table 1. Based on the HRCT, bronchiectasis can be classified into 3 types cylindrical bronchiectasis, varicose bronchiectasis and saccular or cystic bronchiectasis. In cylindrical bronchiectasis the bronchi fail to taper as the bronchi progress peripherally. Varicose bronchiectasis has an irregular and beaded appearance and appears as "a string of pearls". Saccular or cystic bronchiectasis appears as a group of cysts without recognizable bronchial structures distal to the sacs (Webb, WR, High resolution CT chest of the Lung 2009).

\begin{tabular}{|c|c|}
\hline Post-Infectious & Inhalational and Obstruction \\
\hline Lower Respiratory Tract & Severe Gastroesophageal Reflux \\
\hline Granulomatous Infections & Disease \\
\hline Nectrotizing Pneumonias & Chronic Aspiration Pneumonia \\
\hline Other Respiratory infections & Toxic or Thermal Inhalational Injury \\
\hline $\begin{array}{l}\text { Primary Immune Disorders } \\
\text { Hypogammaglobulinemia } \\
\text { Waldendstrom's } \\
\text { Other Humoral / Cellular Disorders } \\
\text { Neutrophil abnormalities }\end{array}$ & $\begin{array}{l}\text { Cystic Fibrosis } \\
\text { Young's Syndrome } \\
\text { Alpha1-Antitrypsin Deficiency }\end{array}$ \\
\hline $\begin{array}{l}\text { Heritable Structural Abnormalities } \\
\text { Ciliated epithelium such as Primary } \\
\text { Ciliary Diskinesia } \\
\text { Cartilage or Connective Tissue } \\
\text { (Tracheobronchiomegaly; Williams- } \\
\text { Campbell) } \\
\text { Sequestration, agenesis, hypoplasia }\end{array}$ & $\begin{array}{l}\text { Allergic Bronchopulmonary } \\
\text { Aspergillosis } \\
\text { Or Other Mycosis } \\
\text { Post-obstruction } \\
\text { Foreign body } \\
\text { Tumor (benign and malignant) }\end{array}$ \\
\hline $\begin{array}{l}\text { Idiopathic Inflammatory Disorders } \\
\text { Sarcoidosis } \\
\text { Rheumatoid Arthritis } \\
\text { Systemic Lupus Erythematosis } \\
\text { Sjogren's Syndrome } \\
\text { Inflammatory Bowel Disease } \\
\text { Relapsing polychondritis }\end{array}$ & $\begin{array}{l}\text { Miscellaneous } \\
\text { HIV infection / AIDS } \\
\text { Yellow Nail Syndrome } \\
\text { Radiation Injury }\end{array}$ \\
\hline
\end{tabular}

Table 1. Differential Diagnosis of Known Etiologies of Bronchiectasis

Patients with bronchiectasis typically present with chronic cough recurrent infections and sputum production (Morrissey 2007). Recurrent infections lead to further airway inflammation and damage which worsens the condition. Other presentations include hemoptysis, chronic airflow obstruction, and slow progressive shortness of breath or dyspnea. Physical examination is variable based on the etiology. Bronchography was used in the past for visualization of bronchiectatic airways. This involved coating the airways with a radiopaque dye instilled through a catheter or a bronchoscope. However, with the advent of HRCT this procedure is rarely being utilized. Pulmonary function tests may show airflow obstruction. 


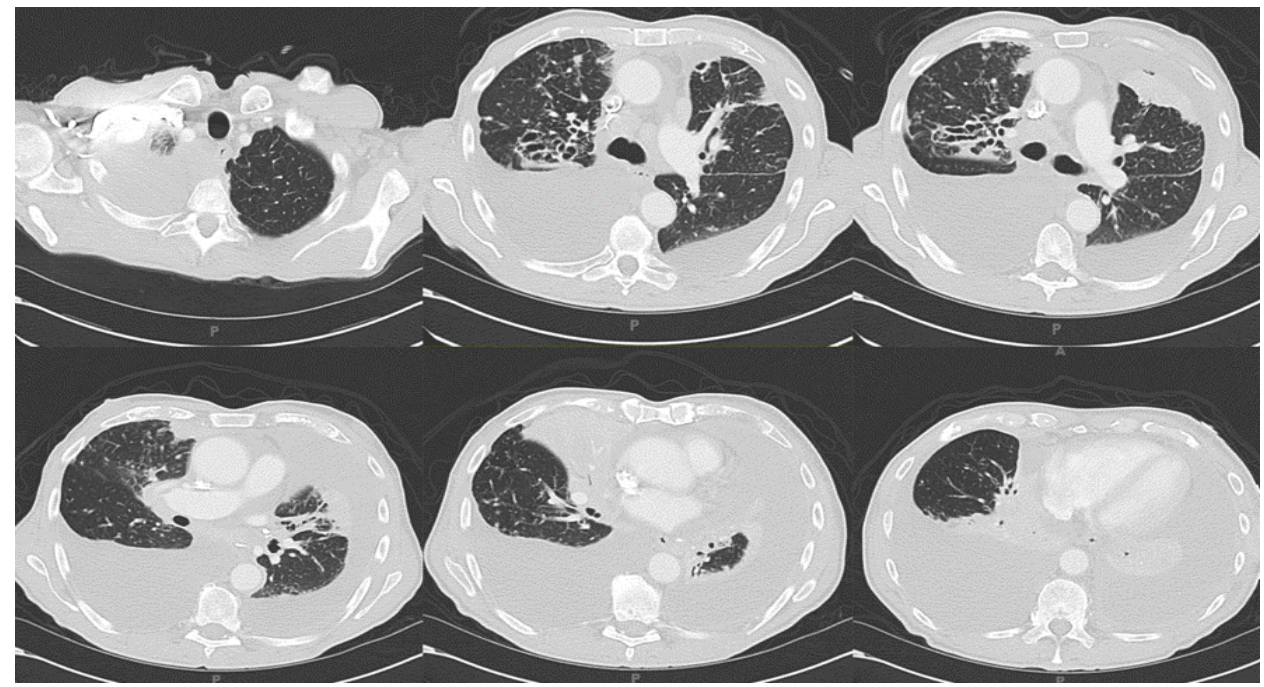

Fig. 1. CT images of a patient with Yellow Nail Syndrome with pleural effusion. Yellow Nail Syndrome is characterized by slow growing curved thickened yellow nails with lymphedema, pleural effusion and bronchiectasis on Computed Tomography. Note the bronchiectatic airways in the right middle lobe.
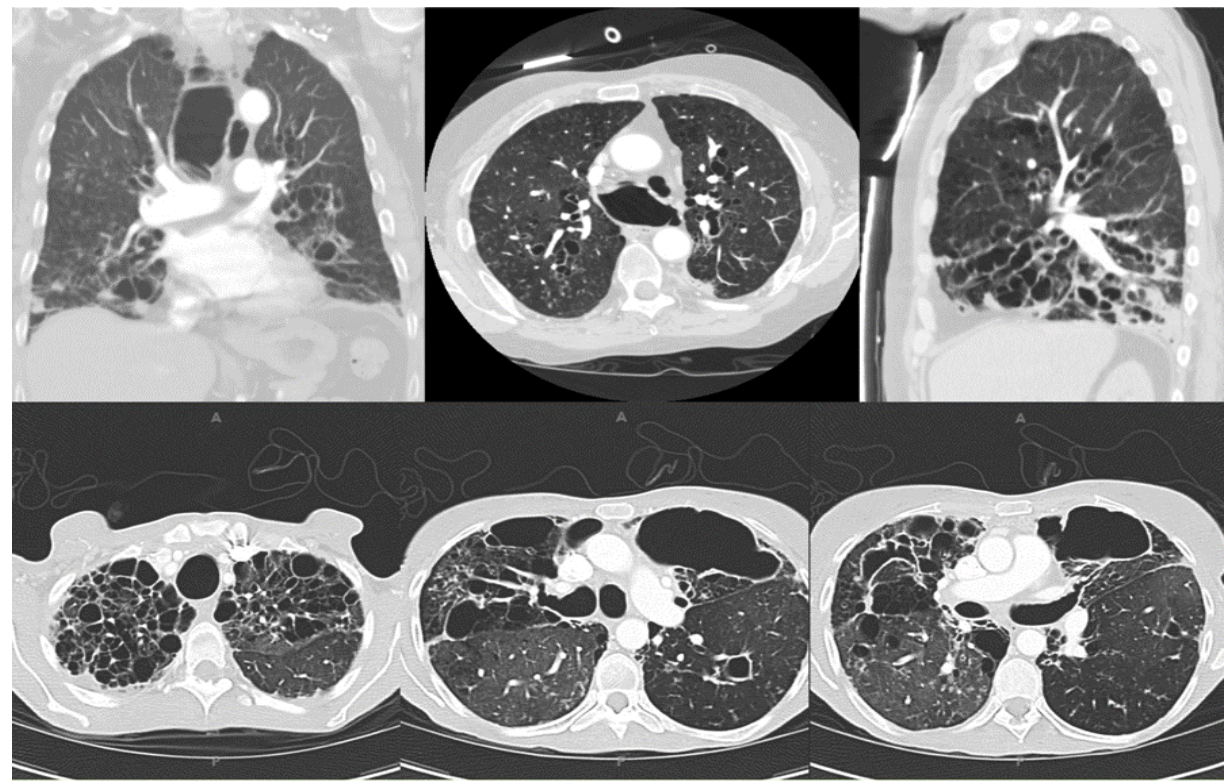

Fig. 2. Tracheobroncheomegaly or Mounier-Kuhn Syndrome. Images show marked dilatation of the trachea and the main stem bronchi. It is characterized by tracheal diameter greater than $3 \mathrm{~cm}$ that is measured $2 \mathrm{~cm}$ above the aortic arch. The right main bronchus should be greater than $2.4 \mathrm{~cm}$ and the left main bronchus greater than $2.3 \mathrm{~cm}$. 


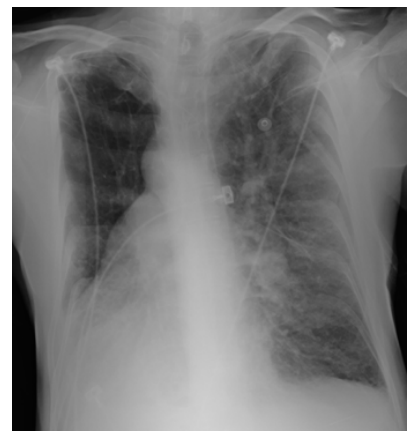

Fig. 3. Plain radiograph of Kartagener's Syndrome, characterized by situs inversus, azospermia, and bronchiectasis.

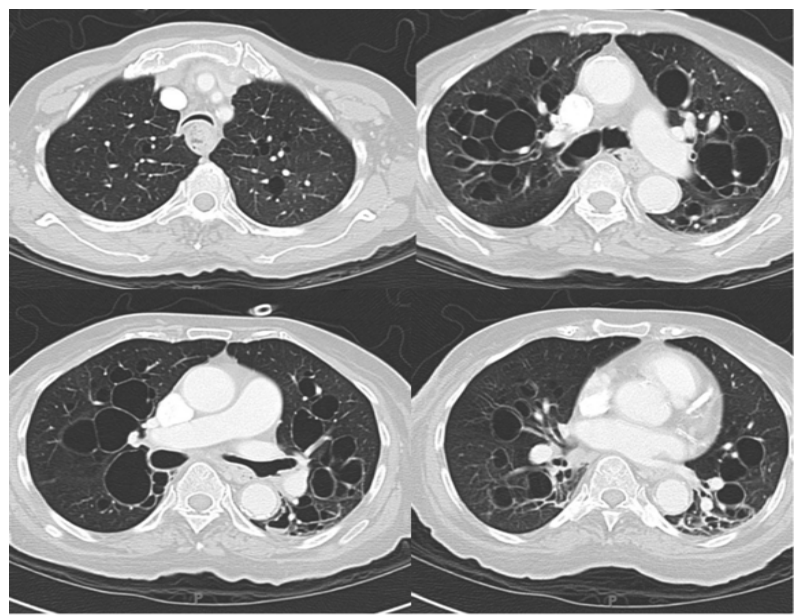

Fig. 4. CT cross sections of a patient with Williams-Campbell syndrome which show bilateral cystic bronchiectasis distal to the third-generation bronchi with abnormal distal lucency probably due to air trapping hyperinflation of lung or bronchiolitis

\section{Overview of Cystic Fibrosis}

Cystic Fibrosis is the most common life-shortening genetic disease of Caucasians affecting more than 25,000 individuals in the United States. Cystic Fibrosis is an autosomal recessive disease due to alteration in the function of a chloride channel; the Cystic Fibrosis Transmembrane Conductance Regulator (CFTR) (Rowe S. M. 2005). In the setting of CFTR dysfunction, an inability of chloride to pass through the channel in the apical membrane of epithelial cells leads to less hydrated secretions from exocrine glands. In the lungs, this causes airway secretions to be thick and difficult to clear. In this milieu, inflammatory cells and colonizing bacteria clogging the airways leads to destruction and dilation with subsequent development of bronchiectasis. The bronchiectasis in patients with Cystic Fibrosis is classically an upper lobe process. However, over time, the destruction becomes widespread involving all lobes. 
In addition to the development of bronchiectasis in individuals with Cystic Fibrosis through the pathophysiology of CFTR dysfunction, coincident bacterial and fungal colonization and infection propagate the airway dilation. Patients with Cystic Fibrosis are often colonized with atypical Mycobacterium (Whittaker, L. A 2009). Mycobacterium avium intracellulare is the most common such infection which leads to bronchiectasis in middle lobe and lingular segments. Moreover, Allergic Bronchopulmonary Aspergillosis affects up to $10 \%$ of CF patients leading to worsening central bronchiectasis (Laufer, P 1984, Knutsen A. P. 2011). The role of bronchoscopy in Cystic Fibrosis will be discussed herein.

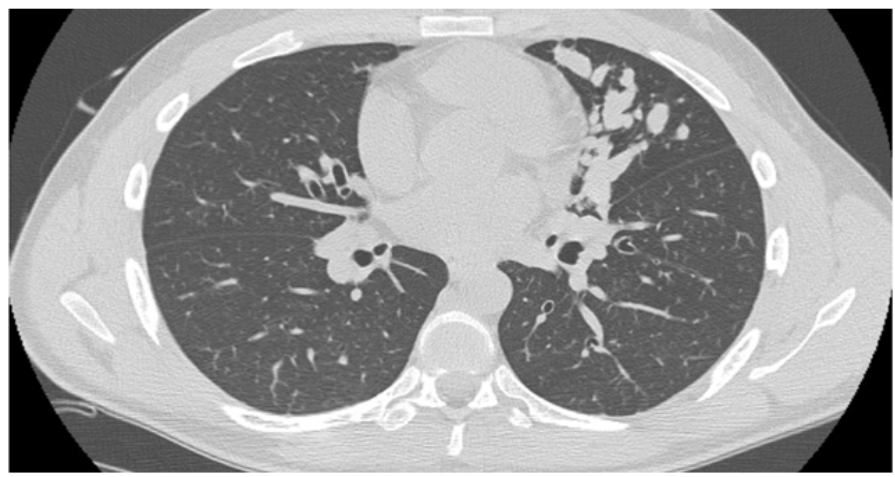

Fig. 5. CT image of right middle lobe involvement of Allergic Bronchopulmonary Aspergillosis with mucus impaction in a patient with Cystic Fibrosis.

\section{Role of bronchoscopy}

\subsection{Role of diagnostic bronchoscopy in bronchiectasis}

Bronchoscopy is not routinely indicated for the diagnosis of bronchiectasis. With the increasing resolution and availability of high-resolution computed tomography (HRCT) scanning, the utility of bronchoscopy in patients with bronchiectasis is not clear, although there exist certain situations in which bronchoscopy provides vital information.

Bronchoscopy for localized bronchiectasis is indicated to evaluate for the presence of an obstructing lesion causing distally located bronchiectasis when lobar or segmental bronchiectasis is apparant on CT imaging. For example, Middle Lobe Syndrome refers to the chronic collapse of the middle lobe which is often associated with distal middle lobe bronchiectasis. The etiology of Middle Lobe Syndrome has both infectious and non infectious causes. It can be divided into obstructive and non obstructive types (Albo, R.J. 1966, Wagner RB 1983, Einarsson, J.T. 2009, ). Bronchoscopy is indicated in all patients with Middle Lobe Syndrome to evaluate for a compressing or obstructing lesion as malignancy is a common cause. (Albo, R.J. 1966, Wagner R.B. 1982, Priftis, K.N. 2005,). Foriegn body may not be apparant on HRCT. Dikensoy et al in their review of foreign body aspiration reported cases of foreign body seen at bronchoscopy which was not evident on CT of the chest (Dikensoy, O. 2002). Cytological examination of bronchoscopic specimens can provide evidence supporting gastric aspiration as a cause of bronchiectasis in dependent lung segments(Pasteur, M.C. 2010). Li et al showed 3/4 cases of bronchiectasis thought to be due to aspiration had lipid-laden macrophages on cytology (Li, A.M. 2005) 
The British Thoracic Society published guidelines related to the utility of bronchoscopy in non-Cystic Fibrosis Bronchiectasis in 2010, which lists several indications for diagnostic bronchoscopy (Pasteur, M.C. 2010). Table 2.

Pasteur et al identified a study by Fernald at el in 1978 in stable state in which bronchoscopy did not show any advantage over sputum culture at identifying lower respiratory tract pathogens (Pasteur, M.C. 2010 Fernald G.W., 1978). Shiekh et al retrospectively evaluated the occurence of bronchiectasis in children with HIV pneumopathy (Shiekh, S. 1997 ). They found that bronchoalveolar lavage (BAL) had a high yield of clinically relevant information that required specific treatment. 22/57 (38.6\%) were positive of Pneumocystis carinii, 14/27 (24.5\%) had CMV and 12/27 (21\%) were positive for MAI.

1. Bronchiectasis in a single lobe to exclude a foreign body obstruction especially in children;

2. In adults with localized disease, bronchoscopy may be indicated to exclude proximal obstruction;

3. Bronchoscopy is useful for obtaining microbiological results in patients who are acutely ill;

4. Bronchoscopic sampling of lower respiratory tract secretions may be indicated in patients with bronchiectasis in whom serial sputum testing is not yielding results and who are worsening;

5. Bronchoscopy can be used to obtain endobronchial biopsy of airway cilia to investigate causes of bronchiectasis

6. Bronchoscopy can be used to localize the site of bleeding in patients with bronchiectasis and hemoptysis which can guide further interventional therapies.

Table 2. British Thoracic Society Guidelines on non-Cystic Fibrosis Bronchiectasis

Pang et al while evaluating the bacteriology of bronchiectasis in Hong Kong, concluded that BAL and protected specimen brush are comparably sensitive in detecting lower respiratory tract organisms (Pang, J.A. 1989). Cabello et al looked at distal airway flora of healthy subjects with chronic lung disease including those with bronchiectasis and found that $88 \%$ of patients had bacterial colonization (Cabello, H. 1997). They also found comparable sensitivities between both BAL and protected specimen brush. Tanaka et al found that bronchial washings was twice as sensitive as expectorated sputum for isolation of Mycobacterium avium complex (Tanaka, E. 1997). They also went on to show that granuloma formation as seen on lung biopsy would suggest infection rather than colonization.

Bronchoscopy in the setting of Tuberculosis is a vast topic and is beyond the scope of this chapter, however, a brief review is warranted. In endemic areas, a positive sputum acid-fast bacilli (AFB) smear is frequently regarded as diagnostic of pulmonary tuberculosis. Negative AFB smears often poses a diagnostic challenge. Tan et al examined the clinical utility of rapid Mycobacterium tuberculosis (MTB) detection in bronchoalveolar lavage (BAL) samples by polymerase chain reaction (PCR). BAL PCR had sensitivity, specificity, positive and negative predictive values of $66.7 \%, 100 \%, 100 \%$ and $88 \%$, respectively, for the group with upper lobe infiltrates (Tan YK 1999). Ismail and coworkers reviewed 232 cases of 
pulmonary tuberculosis and found that $22.8 \%$ of patients had smear positive acid fast bacilli, $11.2 \%$ of patients were diagnosed with tuberculosis based on this. In the setting of negative sputum testing in patients with typical clinical and chest radiographic presentations, the diagnostic accuracy was improved to $49.1 \%$ with bronchoscopic washings. Twenty-four percent of these demonstrated smear positivity and the other $25 \%$ were culture positive (Ismail Y 2004). Watanuki et al. examined 14 subjects with abnormal chest radiographs in which no definite diagnosis could be obtained through sputum analysis including smears and cultures. Bronchoscopic washings were positive for Mycobacterium in 7 patients on smear while cultures were positive in 8 patients. Mycobacterium avium complex (MAC) was identified in 13 patients; however, only $36 \%$ of those tested by specific polymerase chain reaction probes were MAC-positive. In patients undergoing transbronchial lung biopsy $(n=11)$, histological review showed granuloma formation in four and caseation in another three patients (Watanuki Y 1999). Watanabe described 19 cases of bronchial stricture or obliteration due to endobronchial tuberculous lesions. Based on their findings, these authors recommended bronchoscopy and computed tomography as methods of choice for accurate diagnosis of tuberculous bronchial involvement (Watanabe Y 1997). Moreover, these modalities may aid in the assessment for surgery. Based on review of the available literature focusing on Tuberculosis or other Mycobacterial infections in the setting of bronchiectasis, bronchoscopy should be considered when diagnosis is uncertain in high-risk patients or when assessment of intervention is necessary.

\subsection{Role of bronchoscopy in Cystic Fibrosis}

Most of the current literature about the use of bronchoscopy in Cystic Fibrosis comes from pediatric experience. In infants with asymptomatic CF and those diagnosed through newborn screening, bronchoscopy is useful in defining bacterial colonization and inflammation in the airways not obtainable through less invasive means (Rosenfeld, M. 2001). The high yield of microbiology, cytology, and $\mathrm{pH}$ probe investigations in newborn infants with CF suggests that invasive surveillance fiber optic bronchoscopy (FOB) should be considered (Stafler, P. 2001). Bronchoscopy has been used for studying inflammatory markers such as Interleukin -8 and nitrite in the lower airway of patients with Cystic Fibrosis (Noah, T.L. 2003, Cetin, I. 2004, Davis, S.D. 2007, Cobanoglu, N. 2010). Bronchoscopy has similarly been used for identification of atypical airway infections. Semi quantitative culture of bronchoalveolar lavage (BAL) fluid was a useful diagnostic tool in CF patients in whom empiric therapy failed (Baughman, R.P. 1997). Dahm et al. showed fiberoptic bronchoscopy to be a superior technique for obtaining BAL samples as compared to rigid bronchoscopy or bronchial washout (Dahm, L.S. 1977). Davis et al. showed that the BAL fluid inflammation as indicated by the percent of recovered neutrophils and Interleukin -8 levels was significantly higher in the area identified as having the greatest disease on a high resolution chest CT (Davis, S.D. 2007). This highlights the potential importance of performing BAL in more than one area in patients with CF. In a retrospective study, Gilchrist et al showed that single-lobe bronchoalveolar lavage is not sufficient in assessing patients with cystic fibrosis for lower airway infection (Gilchrist, F.J. 2011). Studies have also compared sputum versus BAL in CF (Aaron, S.D. 2004). Bronchoscopy has been used to localize the site of bleeding in patients with CF-related hemoptysis, although there is little evidence for its benefit (Flume, P.A. 2010). 


\subsubsection{Role of Bronchoalveolar Lavage (BAL) in bronchiectasis and CF}

There is evidence of early functional structural and pathological changes including bronchiectasis in babies with CF (Linnane, B.M. 2008, Linnane, B.M., Hall G.L.2008). Hillard et al in UK showed that $44 \%$ of asymptomatic children investigated with Bronchoscopy and BAL had a positive culture prior to newborn screening (Hillard, T.N. 2007). While looking at the patient's after newborn screening they found $27 \%$ of asymptomatic patient's had positive BAL cultures. There was evidence of airway inflammation as well. Whether early identification and aggressive treatment of asymptomatic pulmonary infections delays the decline in lung function is a matter of debate and at the present time there are no longitudinal studies. In a retrospective review of bronchoscopies performed in pediatric cystic fibrosis patient's Boogarard et al showed that 28/66 (42\%) of the BAL provided information that had therapeutic consequences (Boogaard, R. 2008). The European Respiratory Society guidelines in 2000 recommended taking one BAL specimen from the most effected lobe or the right middle lobe (de Blic J 2000). This was later changed to two BALs specimens in 2007 (Brennan, S. 2008). In a retrospective review Gilchrist et al found that if they have used the 2000 ERS guidelines of only sampling a lobe they could have missed 26 positive cultures, while if they used the most current or 2007 guidelines for BAL sampling with 2 BALS at 2 different lobes then they would have missed only 12 positive cultures out of 39 (Gilchrist, F.J. 2011).

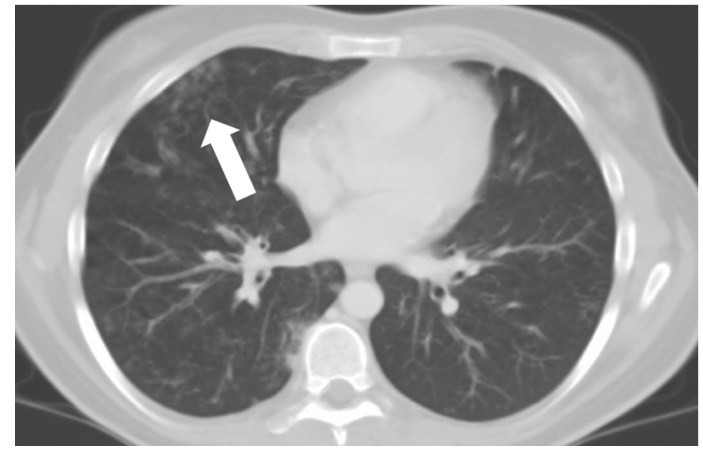

Fig. 6. CT image of a 24-year-old female with Cystic Fibrosis. The patient had frequent pulmonary exacerbations of Cystic Fibrosis and progressive decline in lung function in spite of aggressive appropriate antibiotic therapy guided by expectorated sputum cultures. She subsequently underwent a directed bronchoscopy with BAL in the right middle lobe (arrow) which grew Mycobacterium abscessus. Treatment of this infection broke the cycle of recurrent pulmonary exacerbations.

BAL has also been compared to oropharyngeal swabs to determine the pathogen causing sinus infection by Muhlebach et al. Sinusitis can cause CF exacerbations. The diagnostic accuracy of BAL and oropharyngeal swabs cultures was low in predicting sinus infections especially at younger ages. The positive predictive value of BAL sample for Pseudomonas aeruginosa infection was $65 \%$ while the negative predictive value was $67 \%$. These were similar to oropharyngeal swab cultures. The positive predictive value for Staphylococcus aureus was $76 \%$ and a negative predictive value was $63 \%$ for the BAL fluid. Based on this study both BAL and oropharyngeal swabs are poor predictors of the organisms present in 
the sinus (Muhlebach, M.S. 2006). In the Australasian Cystic Fibrosis Bronchoalveolar Lavage randomized controlled trial cystic fibrosis patients identified through newborn screening were randomly assigned to BAL directed therapy versus standard therapy (Wainwright, C.E. 2011). They reported similar prevalence of Pseudomonas and other organisms amongst the two groups. There were no significant differences in the secondary outcomes such as FEV1, weight or BMI amongst the two groups. BAL fluid in cystic fibrosis patients has also been used to quantify and assess BAL fluid nitrite levels as this may reflect adequate the degree of inflammation in the respiratory tract and could potentially be a useful indicator of airway inflammation for patients with CF (Cetin, I 2004).

Cobanoglu et al studied the levels of ANCA in serum and BAL fluid to identify any relationship with infection (Cobanoglu, N 2010). MacGregor et al analyzed the BAL fluid obtained from CF patients using mass-spectrometry techniques (MacGregor, G 2008).

\subsubsection{Role of protected brushing in bronchiectasis and CF}

Bronchoscopy with protected Brush has been used to collect uncontaminated airway specimen for culture from the lower airways. As the brush is used to directly obtain secretions and bacteria from the airway lumen and then retracted into a sterile sheath, it is thought to have minimal contamination from other airway secretions. Aaron et al (2004) tried to see if bronchoscopy with protected brush would sample biofilm-forming bacteria on the airway wall as opposed to traditional sputum collection techniques. A total of 12 patients were evaluated with protected brush bronchoalveolar lavage and expectorated sputum collection. They showed that 10 patients (83\%) had the same strain of Pseudomonas aeruginosa found using all 3 techniques. They concluded that sputum collection provided as much information for characterization and antibiotic susceptibility testing of Pseudomonas aeruginosa infection as bronchoscopy with protected brush (Aaron, S.D. 2004). As mentioned in previous sections Pang et al as well as Cabello et al showed that protected brush specimen was comparably sensitive to bronchoalveolar lavage in detecting lower respiratory tract organisms in patients with bronchiectasis (Pang, J.A. 1989, Cabello, H. 1997).

\subsubsection{Role of endobronchial and transbronchial biopsy in bronchiectasis and CF}

Endobronchial biopsy during bronchoscopy is a minimally used technique in the diagnostic evaluation of patient's with cystic fibrosis. There is a theoretical concern of bleeding with endobronchial biopsy in these patients due to increased blood flow. Endobronchial biopsy has been used safely in adults with cystic fibrosis during research protocols. Molina-Teran et al retrospectively looked at 45 bronchoscopies with endobronchial biopsies in children with cystic fibrosis at their Institute and matched them to control. They noted 6/45 (13\%) had complications during the procedure of which 4 had significant coughing, 1 had $>10 \%$ drop in oxygen saturation which improved with oxygen and 1 had dental loss. During the 12 hours after the procedure there was no significant complications in both patients with cystic fibrosis and the controls. They did not note any significant bleeding (Molina-Teran, A. 2006). One of the drawback of this study is that they is did not quantify the bleeding. Patients with severe CF were excluded from the study. In 2007 Regamey et al prospectively assessed the quality of the biopsy specimen obtained from children with cystic fibrosis using non-CF patients as control. They concluded that adequate biopsy specimens could be obtained to study the airway of patients with cystic fibrosis (Regamey, N. 2007). They did suggest taking at least 2 biopsies and 
the use of large forceps during the biopsy procedure. As mentioned earlier, in patients with bronchiectasis with suggestion of MAC on HRCT Tanaka et al found that 10/26 who underwent transbronchial lung biopsy had granuloma formation, 8 of whom were positive for MAC and 2 were positive for M. abscessus and M. fortuitum. They showed that this was present with infection and not with colonization (Tanaka, E. 1997).

We can conclude that in the bronchial biopsy can be used safely as a diagnostic tool in the evaluation and management of patient's with Cystic Fibrosis and bronchiectasis. In the setting of uncertainty in CF, it can yield important information leading to therapeutic guidance.

\subsection{Role of bronchoscopy for therapeutics}

Despite the paucity of literature in bronchoscopy for patients with cystic fibrosis and bronchiectasis there are reports of its therapeutic use. Lobar atelectasis is a common complication in patients with cystic fibrosis. Morbidity and mortality of patient's with cystic fibrosis has been linked to their FEV1. As such the consequences of persistent atelectasis in these patients can be serious (Stern, R.C. 1978). Whitaker et al reported a case series of CF patients with Allergic Bronchopulmonary Aspergillosis (ABPA) who successfully underwent sequential bronchoscopy with installation of recombinant human DNase to achieve re-expansion of the lung after they had failed standard medical therapy (Whitaker, P. 2011). McLaughlin et al. successfully treated lobar atelectasis in CF patients with ABPA, which was resistant to conventional therapy with antibiotics and chest physiotherapy, with bronchoscopic instillation of recombinant human deoxyribonuclease (rhDNase) (McLaughlin, A.M. 2008). Slattery et al also showed similar results in another retrospective study. They showed therapeutic bronchoscopy with rhDNase lavage was shown to be safe and associated with improved chest radiographs in pediatric CF patients with persistent atelectasis (Slattery, D.M. 2001).

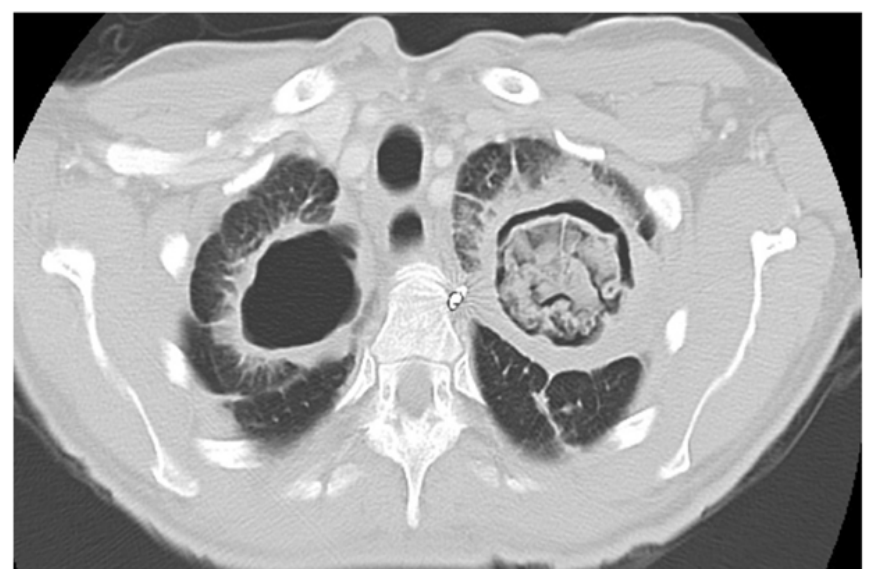

Fig. 7. CT image of a 42-year-old woman with Cystic Fibrosis with recurrent massive hemoptysis. Initially based on imaging, a left-sided source was assumed and she underwent bronchial artery embolization (BAE). As hemoptysis was unremitting, bronchoscopy was performed to localize the source, which was identified and successfully treated with BAE of the right upper lobe vessels. 
Bronchomalacia or collapse of the large airway is only rarely reported as a complication of $\mathrm{CF}$. Herlitz et al. were the first to report the successful deployment of an expandable silicone stent to treat bronchomalcia in a 15 year old with CF (Herlitz, G.N. 2006). This led to clinical improvement as well as improvement in the pulmonary function test obviating the immediate need for lung transplant. Bronchoscopy and transbronchial stenting have been used to treat complications arising from lung transplantation or surgery in patients with cystic fibrosis. Wood at al reported a case of a 20 -year-old with cystic fibrosis who came with recurrent massive hemoptysis that was due to bronchopleural fistula at the stump site. They used superglue (butyl or Methy methacrylate) passed through a Teflon catheter through the working channel of the bronchoscope to seal the stump (Wood, R.E. 1992). Bronchoscopy has been used in management of hemoptysis in patients with CF and bronchiectasis. Joseph at al used bronchoscopic techniques for gene transfer to airway epithelium using adenoviruses for cystic fibrosis (Joseph, P.M. 2001, Perricone, M.A. 2001).

\section{Safety of bronchoscopy in Cystic Fibrosis}

Adverse events are common with BAL in young CF children, but are usually transient and well tolerated (Wainwright, C.E. 2008). Wainwright at al conducted a prospective multicenter study to systematically evaluate safety and adverse events associated with BAL in pediatric CF patients. They looked at pediatric patients undergoing BAL between September 1999 and December 2005. Twenty-nine of 333 (8.7\%) of the BALs were followed by fever; 10 (3\%) had clinically significant deterioration of which five required assisted ventilation during the procedure due to desaturation; one had a ventricular tachyarrhythmia after the use of an anesthetic agent; and one was hospitalized for stridor and respiratory distress. The rates of patients with fever in this study were no different than the rates reported in other studies in children without CF. Transient fever after BAL is thought to be associated with elevated serum pro inflammatory markers and can develop without bacteremia. They did find that focal bronchitis was associated with clinically significant deterioration during or after the BAL procedure, the reason for which were not entirely clear. Another strong association noted was between Streptococcus pneumonia in BAL fluid and fever afterwards. Worsening of cough was the most frequently reported minor event. It did not result in any increase in hospitalization. As mentioned previously, in a retrospective review MolinaTeran et al. showed that endobronchial biopsy performed under general anesthesia can be done safely in children with CF (Molina-Teran, A. 2006). They noted 6/45 (13\%) had complications during the procedure of which 4 had significant coughing, 1 had $>10 \%$ drop in oxygen saturation which improved with oxygen and 1 had dental loss. During the 12 hours after the procedure there was no significant complications in both patients with cystic fibrosis and the controls. They did not note any significant bleeding. The major drawback of this study was that it did not quantify the bleeding or include patients with severe disease. Endobronchial biopsy has been performed safely in adult CF patients for the purpose of research.

\section{Role of anaesthesia and sedation}

As with all procedures, the safety of the patient is paramount. Decisions regarding the best approach for patient control and comfort surround multiple patient factors as well as consideration for the duration and technical aspects of the procedure. Conscious sedation is 
performed by many providers performing bronchoscopies on patients with bronchiectasis and cystic fibrosis. However, due to profound bronchoscopasm often experienced with airway manipulation, consideration for the utilization of anesthesia support should strongly be considered in patients with bronchiectasis and cystic fibrosis.

Little was known initially about the role of general anesthesia in patients with Cystic Fibrosis. Price showed that a marked deterioration in lung function took place after a brief anesthetic for a relatively minor surgical procedure (Price, J.F. 1986). In a retrospective review, Chhajed et al showed that the sedative drug requirements during bronchoscopy were higher in patients with CF, especially after lung transplantation (Chhajed, P.N. 2005). Few authors reviewed the preoperative assessment, intraoperative and postoperative management of patients with CF (Huffmyer, J.L. 2009, Della Rocca, G. 2002, Walsh, T.S. 1995). An anesthetic plan for patients with CF undergoing procedures was described by Karlet et al (Karlet, M.C. 2000).

The respiratory dysfunction associated with cystic fibrosis should be the major focus of preoperative evaluation by the anesthesiologist. The anesthesiologist should quantify the level of pulmonary function and extent of cardiopulmonary limitation preoperatively and attempts must be made to optimize it prior to the procedure. Historically, the amounts of sputum production, decreased exercise tolerance, decreasing weight are important features. If patients report an increased cough, wheezing, increased sputum production, decreased activity level, infection or other symptoms to suggest CF exacerbation, admission to the hospital for optimization of lung function should be considered. Hypercarbia typically develops late in cystic fibrosis.

All cases should receive chest radiograph, pulmonary function testing, serum electrolyte levels including blood glucose levels, complete blood cell count and liver function tests (Della Rocca, G. 2002). Depending on the type of bronchoscopy being performed and the patient's history of liver involvement, further coagulation testing may be ordered. Moreover, arterial blood gas measurements, electrocardiogram, and echocardiogram may also be performed prior to the procedure if warranted by the patient's history.

Patient's routine medications including bronchodilators and corticosteroids should be continued into the perioperative period. Sedatives and analgesics should be used cautiously in the preoperative period as they can depress the respiratory drive and patient's inability to clear secretions (Huffmyer, J.L. 2009). Premedication with an antacid such as H2 blocker should be considered as there is a higher incidence of gastroesophageal reflux (Weeks, A.M. 1995).

Procedural monitoring should include continuous electrocardiography, respiratory rate, oximetry, and blood pressure. Capnography may be beneficial in patients requiring significant sedation or in those with moderate to severe lung disease. Arterial blood gas monitoring should be performed for patients with severe disease. Frequent intraoperative and postoperative management of blood sugars is indicated for prolonged procedures. Ventilating pressures should be monitored carefully as patients with CF are at risk for rupture of emphysematous bullae resulting in a pneumothorax (Karlet, M.C. 2000) in the setting of air trapping.

Preoxygenation to achieve higher oxygen saturation before induction is important. History of significant gastroesophageal reflux would require a rapid sequence induction. The short life of 
Propofol makes it an ideal choice. Ketamine is relatively contraindicated due to increased bronchial secretions. The advantage of volatile inhalational agents used in general anesthesia is that they cause bronchodilation, decrease muscle relaxant dosage, and reduced airway hyperreactivity. Although Desflurane has an undesired pungent odor and respiratory tract irritability which makes it a poor choice of agent. Systemic hydration and humidification of inspired gasses are important for maintaining secretions in a less viscous state. If muscle relaxants are used short-acting nondepolarizing agents may be more appropriate. They should be administered in minimum possible doses. It should be noted that the aminoglycoside antibiotics use to treat infections might prolong the neuromuscular blocking affect. Extubation should be delayed until the effect of muscle relaxants has worn off.

Anesthesia should be tailored towards rapid postoperative recovery of the ability to cough and take deep breaths to actively clear secretions. Post procedure aggressive suctioning, chest physiotherapy and oxygen supplementation should be continued till the effect of the anesthetic agent has worn off and patient is back to his/her previous state of health.

\begin{tabular}{|l|l|}
\hline Suggested tests & Further testing \\
Serum electrolytes & Coagulation tests \\
Complete blood count & Arterial blood gas \\
Liver function tests & Echocardiogram \\
Chest X ray & \\
Pulmonary function tests & \\
Blood glucose levels & \\
Electrocardiogram & \\
\hline
\end{tabular}

Adapted from Karlet, 2002

Table 3. Preoperative test for Anesthesia

\section{Approach to performing bronchoscopy in bronchiectasis and CF}

Preparation for bronchoscopy in patients with cystic fibrosis or chronic suppurative bronchiectasis is similar to the initial evaluation for anaesthesia. Important aspects include a clear understanding of the indication for bronchoscopy. As the pulmonologist understanding the current and usual steady state of the patients' respiratory function is critical. While there are no clear guidelines to support peri-operative antibiotics these patients may have a higher likelihood of transient bacteraemia or post bronchoscopic febrile episodes. Judicious clinical judgement of the patients overall condition should be followed in determining which patients may benefit from peri-operative antibiotics.

Having a clear understanding of the indications for the procedure in these patients is not only important in weighing risk and benefit but in allowing an expeditious procedure. Patients with advanced obstructive lung disease may have difficulty with hypoxemia and other complications. A well planned procedure will shorten the duration and attempt to mitigate risk.

If the indication is for airway sampling for culture, care must be taken to avoid contamination of the cultures by suctioning in the upper airway. This may be difficult due 
to the tenacious sputum and upper airway secretions in patients with cystic fibrosis. This is usually less of an issue in those with suppurative bronchiectasis. Strong consideration should be given to protected brush specimen retrieval in all these patients. Ensuring rapid and appropriate transport to the laboratory and rapid plating will significantly aid in obtaining viable culture specimens.

Patients with chronic suppuration coincident with bronchiectasis may have mucosa that is particularly friable. Having a seasoned bronchoscopist is the best choice to avoid mucosal injury and provide the shortest procedural time. These patients are likely not the best candidates for early trainees in bronchoscopy or those who rarely perform bronchoscopy. When these patients present with haemoptysis localization may be critical to guiding bronchial or intercostals arterial embolization. In addition, visualization of a more proximal bleed can occasionally occur. In these cases endobronchial therapies are warranted. The authors would recommend non contact therapies such as argon plasma coagulation or very low wattage Nd:YAG laser. If the indication for the bronchoscopy is mucosal sampling for ciliary dyskinesias a critical step is ensuring the correct specimen handling procedures are known and strictly followed. Most laboratories do not routinely process specimen's electron micrography. Ensuring good pre-procedural communication cannot be understated.

With the advancing population age of cystic fibrosis patients and the presence of bronchiectasis in middle age or older individuals the potential of central airway obstruction must be considered. Both primary bronchogenic carcinoma and metastatic airway disease may occur. Therapeutic bronchoscopy should be considered for these patients if central airway obstruction is due to endobronchial tumor. Therapeutic decision-making, such as risk to benefit of placing a endobronchial foreign body in a suppurative airway makes these cases extremely challenging. The authors would recommend referral to an interventional pulmonologist in a "center of excellence" to provide the best care for these complicated patients. Endoluminal ablative therapies are not contraindicated in these patients but experience is fairly limited. A center with a high volume of therapeutic bronchoscopy is likely the best choice.

\section{Conclusions}

Bronchoscopy provides useful diagnostic information in patients with bronchiectasis and Cystic Fibrosis. The use of these techniques aids the clinician in preventing the considerable morbidity associated with these conditions, and a proper understanding of the intricices of bronchoscopic procedures in these patients is paramount to success.

\section{References}

Aaron, S. D., D. Kottachchi, et al. (2004). "Sputum versus bronchoscopy for diagnosis of Pseudomonas aeruginosa biofilms in cystic fibrosis." Eur Respir J 24(4): 631-637.

Albo, R. J. and O. F. Grimes (1966). "The middle lobe syndrome: a clinical study." Dis Chest 50(5): 509-518.

Barker, A. F. (2002). "Bronchiectasis." N Engl J Med 346(18): 1383-1393.

Baughman, R. P., D. A. Keeton, et al. (1997). "Use of bronchoalveolar lavage semiquantitative cultures in cystic fibrosis." Am J Respir Crit Care Med 156(1): 286291. 
Boogaard, R., et al., Yield from Flexible Bronchoscopy in Pediatric Cystic Fibrosis Patients. J Bronchol, 2008. 15(4): p. 240-246

Brennan, S., C. Gangell, et al. (2008). "Disease surveillance using bronchoalveolar lavage." Paediatr Respir Rev 9(3): 151-159.

Cabello, H., A. Torres, et al. (1997). "Bacterial colonization of distal airways in healthy subjects and chronic lung disease: a bronchoscopic study." Eur Respir J 10(5): 11371144 .

Cetin, I., U. Ozcelik, et al. (2004). "BALF nitrite as an indicator of inflammation in children with cystic fibrosis." Respiration 71(6): 625-629.

Chang, A. B., N. C. Boyce, et al. (2002). "Bronchoscopic findings in children with non-cystic fibrosis chronic suppurative lung disease." Thorax 57(11): 935-938.

Chhajed, P. N., C. Aboyoun, et al. (2005). "Sedative drug requirements during bronchoscopy are higher in cystic fibrosis after lung transplantation." Transplantation 80(8): 10811085.

Cobanoglu, N., U. Ozcelik, et al. (2010). "Anti-neutrophil cytoplasmic antibodies (ANCA) in serum and bronchoalveolar lavage fluids of cystic fibrosis patients and patients with idiopathic bronchiectasis." Turk J Pediatr 52(4): 343-347.

Cohen, M. and S. A. Sahn (1999). "Bronchiectasis in systemic diseases." Chest 116(4): 10631074.

Dahm, L. S., C. W. Ewing, et al. (1977). "Comparison of three techniques of lung lavage in patients with cystic fibrosis." Chest 72(5): 593-596.

Davis, S. D., L. A. Fordham, et al. (2007). "Computed tomography reflects lower airway inflammation and tracks changes in early cystic fibrosis." Am J Respir Crit Care Med 175(9): 943-950.

de Blic, J., F. Midulla, et al. (2000). "Bronchoalveolar lavage in children. ERS Task Force on bronchoalveolar lavage in children. European Respiratory Society." Eur Respir J 15(1): 217-231.

Della Rocca, G., Anaesthesia in patients with cystic fibrosis. Curr Opin Anaesthesiol, 2002. 15(1): p. 95-101.

Dikensoy, O., C. Usalan, et al. (2002). "Foreign body aspiration: clinical utility of flexible bronchoscopy." Postgrad Med J 78(921): 399-403.

Einarsson, J. T., J. G. Einarsson, et al. (2009). "Middle lobe syndrome: a nationwide study on clinicopathological features and surgical treatment." Clin Respir J 3(2): 77-81.

Fernald, G. W. (1978). "Bronchiectasis in childhood: a 10-year survey of cases treated at North Carolina Memorial Hospital." N C Med J 39(6): 368-372.

Flume, P. A., P. J. Mogayzel, Jr., et al. (2010). "Cystic fibrosis pulmonary guidelines: pulmonary complications: hemoptysis and pneumothorax." Am J Respir Crit Care Med 182(3): 298-306.

Gilchrist, F. J., S. Salamat, et al. (2011). "Bronchoalveolar lavage in children with cystic fibrosis: how many lobes should be sampled?" Arch Dis Child 96(3): 215-217.

Herlitz, G. N., D. I. Sternberg, et al. (2006). "Treatment of bronchomalacia in cystic fibrosis by silicone stent." Ann Thorac Surg 82(6): 2268-2270.

Hilliard, T. N., S. Sukhani, et al. (2007). "Bronchoscopy following diagnosis with cystic fibrosis." Arch Dis Child 92(10): 898-899.

Huffmyer, J. L., K. E. Littlewood, et al. (2009). "Perioperative management of the adult with cystic fibrosis." Anesth Analg 109(6): 1949-1961. 
Ismail, Y. (2004). "Pulmonary tuberculosis--a review of clinical features and diagnosis in 232 cases." Med J Malaysia 59(1): 56-64.

Joseph, P. M., B. P. O'Sullivan, et al. (2001). "Aerosol and lobar administration of a recombinant adenovirus to individuals with cystic fibrosis. I. Methods, safety, and clinical implications." Hum Gene Ther 12(11): 1369-1382.

Karlet, M. C. (2000). "An update on cystic fibrosis and implications for anesthesia." AANA J 68(2): 141-148.

Knutsen, A. P. and R. G. Slavin (2011). "Allergic bronchopulmonary aspergillosis in asthma and cystic fibrosis." Clin Dev Immunol 2011: 843763.

Laufer, P., J. N. Fink, et al. (1984). "Allergic bronchopulmonary aspergillosis in cystic fibrosis." J Allergy Clin Immunol 73(1 Pt 1): 44-48.

Li, A. M., S. Sonnappa, et al. (2005). "Non-CF bronchiectasis: does knowing the aetiology lead to changes in management?" Eur Respir J 26(1): 8-14.

Linnane, B., P. Robinson, et al. (2008). "Role of high-resolution computed tomography in the detection of early cystic fibrosis lung disease." Paediatr Respir Rev 9(3): 168-174; quiz 174-165.

Linnane, B. M., G. L. Hall, et al. (2008). "Lung function in infants with cystic fibrosis diagnosed by newborn screening." Am J Respir Crit Care Med 178(12): 1238-1244.

MacGregor, G., R. D. Gray, et al. (2008). "Biomarkers for cystic fibrosis lung disease: application of SELDI-TOF mass spectrometry to BAL fluid." J Cyst Fibros 7(5): 352358.

McLaughlin, A. M., E. McGrath, et al. (2008). "Treatment of lobar atelectasis with bronchoscopically administered recombinant human deoxyribonuclease in cystic fibrosis?" Clin Respir J 2(2): 123-126.

Molina-Teran, A., T. N. Hilliard, et al. (2006). "Safety of endobronchial biopsy in children with cystic fibrosis." Pediatr Pulmonol 41(11): 1021-1024.

Muhlebach, M. S., M. B. Miller, et al. (2006). "Are lower airway or throat cultures predictive of sinus bacteriology in cystic fibrosis?" Pediatr Pulmonol 41(5): 445-451.

Murray, J.F., Nadal, J.A. (2010) Murray E Nadal's Textbook of Respiratory Medicine (5 ${ }^{\text {th }}$ Edition) Saunders, Elsevier, ISBN: 978-1-4160-47100-0, Philadelphia, USA

Noah, T. L., P. C. Murphy, et al. (2003). "Bronchoalveolar lavage fluid surfactant protein-A and surfactant protein-D are inversely related to inflammation in early cystic fibrosis." Am J Respir Crit Care Med 168(6): 685-691.

O'Donnell, A. E. (2008). "Bronchiectasis." Chest 134(4): 815-823.

Pang, J. A., A. Cheng, et al. (1989). "The bacteriology of bronchiectasis in Hong Kong investigated by protected catheter brush and bronchoalveolar lavage." Am Rev Respir Dis 139(1): 14-17.

Pasteur, M. C., D. Bilton, et al. (2010). "British Thoracic Society guideline for non-CF bronchiectasis." Thorax 65 Suppl 1: i1-58.

Perricone, M. A., J. E. Morris, et al. (2001). "Aerosol and lobar administration of a recombinant adenovirus to individuals with cystic fibrosis. II. Transfection efficiency in airway epithelium." Hum Gene Ther 12(11): 1383-1394.

Price, J. F. (1986). "The need to avoid general anaesthesia in cystic fibrosis." J R Soc Med 79 Suppl 12: 10-12.

Priftis, K. N., D. Mermiri, et al. (2005). "The role of timely intervention in middle lobe syndrome in children." Chest 128(4): 2504-2510. 
Quast, T. M., A. R. Self, et al. (2008). "Diagnostic evaluation of bronchiectasis." Dis Mon 54(8): 527-539.

Regamey, N., T. N. Hilliard, et al. (2007). "Quality, size, and composition of pediatric endobronchial biopsies in cystic fibrosis." Chest 131(6): 1710-1717.

Rosenfeld, M., R. L. Gibson, et al. (2001). "Early pulmonary infection, inflammation, and clinical outcomes in infants with cystic fibrosis." Pediatr Pulmonol 32(5): 356-366.

Rowe, S. M., S. Miller, et al. (2005). "Cystic fibrosis." N Engl J Med 352(19): 1992-2001.

Sheikh, S., K. Madiraju, et al. (1997). "Bronchiectasis in pediatric AIDS." Chest 112(5): 12021207.

Slattery, D. M., D. A. Waltz, et al. (2001). "Bronchoscopically administered recombinant human DNase for lobar atelectasis in cystic fibrosis." Pediatr Pulmonol 31(5): 383388.

Stafler, P., J. C. Davies, et al. (2011). "Bronchoscopy in Cystic Fibrosis Infants Diagnosed by Newborn Screening." Pediatr Pulmonol.

Stern, R. C., T. F. Boat, et al. (1978). "Treatment and prognosis of lobar and segmental atelectasis in cystic fibrosis." Am Rev Respir Dis 118(5): 821-826.

Tan, Y. K., A. S. Lee, et al. (1999). "Rapid mycobacterial tuberculosis detection in bronchoalveolar lavage samples by polymerase chain reaction in patients with upper lobe infiltrates and bronchiectasis." Ann Acad Med Singapore 28(2): 205-208.

Tanaka, E., R. Amitani, et al. (1997). "Yield of computed tomography and bronchoscopy for the diagnosis of Mycobacterium avium complex pulmonary disease." Am J Respir Crit Care Med 155(6): 2041-2046.

Wagner, R. B. and M. R. Johnston (1983). "Middle lobe syndrome." Ann Thorac Surg 35(6): 679-686.

Wainwright, C. E., K. Grimwood, et al. (2008). "Safety of bronchoalveolar lavage in young children with cystic fibrosis." Pediatr Pulmonol 43(10): 965-972.

Wainwright, C.E.,S. Vidmar, et al. (2011). " Effect of Bronchoalveolar Lavage-directed therapy on Pseudomonas aeruginosa infection and structural lung injury in children with Cystic Fibrosis: A randomized trial ." JAMA 306(2):163-171.

Walsh, T.S. and C.H. Young, Anaesthesia and cystic fibrosis. Anaesthesia, 1995. 50(7): p. 61422.

Watanabe, Y., S. Murakami, et al. (1997). "Treatment of bronchial stricture due to endobronchial tuberculosis." World J Surg 21(5): 480-487.

Watanuki, Y., S. Odagiri, et al. (1999). "Usefulness of bronchoscopy for the diagnosis of atypical pulmonary mycobacteriosis." Kansenshogaku Zasshi 73(8): 728-733.

Webb, W.R. (2009) High Resolution CT of the lung (4th Edition) Lippincott Williams \& Wilkins, ISBN-13: 978-0-7817-6909-4, Philadelphia, USA

Weeks, A.M. and M.R. Buckland, Anaesthesia for adults with cystic fibrosis. Anaesth Intensive Care, 1995. 23(3): p. 332-8.

Weycker, D., J. Edelsberg, et al. (2005). "Prevalence and Economic Burden of Bronchiectasis." Clinical Pulmonary Medicine 12(4): 205-209.

Whitaker, P., K. Brownlee, et al. (2011). "Sequential Bronchoscopy in the Management of Lobar Atelectasis Secondary to Allergic Bronchopulmonary Aspergillosis." Journal of Bronchology \& Interventional Pulmonology 18(1): 57-60

Whittaker, L. A. and C. Teneback (2009). "Atypical mycobacterial and fungal infections in cystic fibrosis." Semin Respir Crit Care Med 30(5): 539-546. 
Wood, R. E., S. R. Lacey, et al. (1992). "Endoscopic management of large, postresection bronchopleural fistulae with methacrylate adhesive (Super Glue)." J Pediatr Surg 27(2): 201-202. 


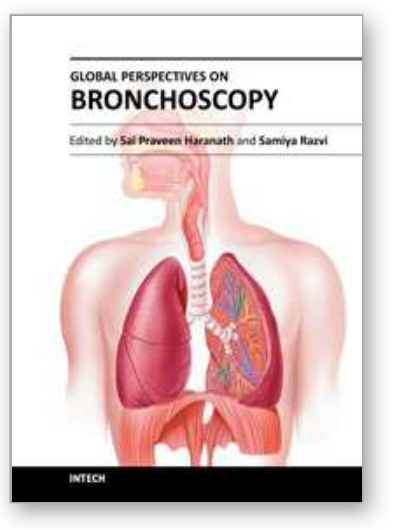

\author{
Global Perspectives on Bronchoscopy \\ Edited by Dr. Sai P. Haranath
}

ISBN 978-953-51-0642-5

Hard cover, 240 pages

Publisher InTech

Published online 13, June, 2012

Published in print edition June, 2012

Bronchoscopy has become an essential part of modern medicine. Recent advances in technology have allowed integration of ultrasound with this tool. The use of lasers along with bronchoscopes has increased the therapeutic utility of this device. Globally an increasing number of pulmonary specialists, anaesthesiologists and thoracic surgeons are using the bronchoscope to expedite diagnosis and treatment. The current volume on bronchoscopy adds to the vast body of knowledge on this topic. The democratic online access to this body of knowledge will greatly increase the ease with which both trainees and expert bronchoscopists can learn more. The contributions from around the world cover the breadth of this field and includes cutting edge uses as well as a section on pediatric bronchoscopy. The book has been an effort by excellent authors and editors and will surely be a often reviewed addition to your digital bookshelf. . In summary, this book is a great testament to the power of collaboration and is a superb resource for doctors in training, ancillary team members as well as practicing healthcare providers who have to perform or arrange for bronchoscopy or the associated procedures.

\title{
How to reference
}

In order to correctly reference this scholarly work, feel free to copy and paste the following:

Aditya Kasarabada, Mark E. Lund and Jeffrey B. Hoag (2012). Bronchoscopy in Bronchiectasis and Cystic Fibrosis, Global Perspectives on Bronchoscopy, Dr. Sai P. Haranath (Ed.), ISBN: 978-953-51-0642-5, InTech, Available from: http://www.intechopen.com/books/global-perspectives-on-bronchoscopy/bronchoscopy-inbronchiectasis-and-cystic-fibrosis

\section{INTECH}

open science | open minds

\section{InTech Europe}

University Campus STeP Ri

Slavka Krautzeka 83/A

51000 Rijeka, Croatia

Phone: +385 (51) 770447

Fax: +385 (51) 686166

www.intechopen.com

\section{InTech China}

Unit 405, Office Block, Hotel Equatorial Shanghai

No.65, Yan An Road (West), Shanghai, 200040, China 中国上海市延安西路65号上海国际贵都大饭店办公楼 405 单元

Phone: +86-21-62489820

Fax: $+86-21-62489821$ 
(C) 2012 The Author(s). Licensee IntechOpen. This is an open access article distributed under the terms of the Creative Commons Attribution 3.0 License, which permits unrestricted use, distribution, and reproduction in any medium, provided the original work is properly cited. 